\title{
Mass-Energy-Momentum: Only there because of Spacetime?
}

\author{
Dennis Lehmkuhl, \\ Oriel College, Oxford University; and \\ IZWT, University of Wuppertal \\ Email: dennis.lehmkuhl@uni-wuppertal.de
}

\begin{abstract}
I describe how relativistic field theory generalises the paradigm property of material systems, the possession of mass, to the requirement that they have a mass-energy-momentum density tensor $T_{\mu \nu}$ associated with them. I argue that $T_{\mu \nu}$ does not represent an intrinsic property of matter. For it will become evident that the definition of $T_{\mu \nu}$ depends on the metric field $g_{\mu \nu}$ in a variety of ways. Accordingly, since $g_{\mu \nu}$ represents the geometry of spacetime itself, the properties of mass, stress, energy and momentum should not be seen as intrinsic properties of matter, but as relational properties that material systems have only in virtue of their relation to spacetime structure.
\end{abstract}

\section{Contents}

1 Introduction $\quad 2$

$\begin{array}{lll}2 & \text { The concept of matter in field theory } & \mathbf{6}\end{array}$

2.1 A short history of energy-momentum tensors . . . . . . . . 7

2.2 Metaphysical matters . . . . . . . . . . . . . . . 12

3 Explicit metric dependence 12

4 Metric dependence in Lagrangian theories 14

4.1 The basic ideas of Lagrangian field theory . . . . . . . . . . . 14

4.2 Enter the energy tensor . . . . . . . . . . . . . . 16

4.3 Different kinds of coupling . . . . . . . . . . . . 18 
5 Metric dependence in general 21

5.1 Definitional dependence at the level of the matter fields . . . . 23

5.2 Definitional dependence at the level of conditions on the energy tensor . . . . . . . . . . . . . . . . 25

5.3 Abstract definitional dependence . . . . . . . . . . 26

5.4 Interpretational dependence . . . . . . . . . . . . . . 27

6 What kind of property is mass-energy-momentum? 30

6.1 A relational property? . . . . . . . . . . . . . . 30

6.2 Relational and essential? . . . . . . . . . . . . . . 33

7 Conclusion 35

\section{Introduction}

In the Stanford Encyclopaedia for Philosophy, in the entry "Intrinsic and Extrinsic properties", Weatherson [2007] writes:

I have some of my properties purely in virtue of the way I am. (My mass is an example.) I have other properties in virtue of the way I interact with the world. (My weight is an example.) The former are the intrinsic properties, the latter are the extrinsic properties.

The claim that mass is intrinsic is initially plausible, whereas properties like velocity, momentum and consequently energy - or at least kinetic energy seem paradigm cases of extrinsic properties. A potential problem arises in relativity theory (special and general), where the concepts of mass, energy, momentum and their respective densities and current densities are described by a single mathematical object, the mass-stress-energy-momentum density tensor $T_{\mu \nu}$. The question is then whether one should regard $T_{\mu \nu}$ as representing an intrinsic or an extrinsic property (or set of properties) of material systems. ${ }^{1}$

\footnotetext{
${ }^{1}$ I shall address the historical question of how mass density, energy density and momentum density were summarised into a single tensor $T_{\mu \nu}$ in section 2.1. Conceptually, the question whether $T_{\mu \nu}$ should be interpreted as a single property or as a set of properties seems similar to asking whether the electromagnetic field tensor $F_{\mu \nu}$ represents one single property (electromagnetic field strength) or two (electric field strength and magnetic
} 
I shall argue that mass-energy-momentum density, as described in relativity theory, is not an intrinsic property of material systems, but a property they have only in virtue of their relation to spacetime structure, in particular to the metric tensor $g_{\mu \nu}$. Nevertheless, the non-vanishing of a mass-energymomentum density tensor $T_{\mu \nu}$ at a point will turn out to be a necessary and sufficient condition for this point to be occupied by matter. ${ }^{2}$

It will emerge that the energy tensor $T_{\mu \nu}$ is in important ways less fundamental than the metric field $g_{\mu \nu}$. Historically, Mach's principle, the idea that the reverse was the case, was very important for Einstein up until 1921. In Einstein [1918], p.38, he expresses the principle in the following way: ${ }^{3}$

Mach's principle: The $\left[g_{\mu \nu}\right]$-field is fully determined by the masses

field strength). In the case of the Faraday tensor the answer seems clear: the question of whether electric or magnetic fields are present depends on the frame of reference; hence electromagnetic field strength should be seen as the fundamental property. One might wish to make a similar case for the different components of $T_{\mu \nu}$, arguing that massenergy-momentum density should be seen as the (single) fundamental property. However, it should be noted that Lange [2001, 2002] and Flores [2005] have recently revived discussion of what the 'equivalence' between mass $m$ and energy $E$ of a material system really means, both authors attacking the intuition that 'equivalence' should be understood as 'identity', while drawing different conclusions with regard to how 'equivalence' should be understood. Both authors, though, merely investigate the question of what $E=m c^{2}$ means in the context of special relativistic particle mechanics. In order to give a conclusive answer it seems necessary to extend their discussion to more general material systems characterised by a complete $T_{\mu \nu}$. It seems plausible that in this more general case we might find ourselves forced to accept that there is just a single property, mass-energy-momentum density. At any rate, the main point of this article is independent of the answer to the question of whether $T_{\mu \nu}$ should be seen as representing only one property or a set of properties. In the first case, my argumentation would be interpreted as concluding that $T_{\mu \nu}$ represents a relational property; in the second that it represents a set of relational properties.

${ }^{2}$ For simplicity, I shall often just speak of the 'energy-momentum tensor' or even just of the 'energy tensor' of a material system, rather than of a mass-stress-energy-momentum density tensor. Note that $T_{\mu \nu}$ is not a tensor density in the mathematical sense: like the scalar field $\rho$ in Newtonian theory, it is a tensor that represents a physical density, rather than a mathematical object that transforms as a tensor density.

3 "Machsches Prinzip: Das G-Feld ist restlos durch die Massen der Körper bestimmt. Da Masse und Energie nach den Ergebnissen der speziellen Relativitätstheorie das Gleiche sind und die Energie formal durch den symmetrischen Energietensor $\left(T_{\mu \nu}\right)$ beschrieben wird, so besagt dies, dass das G-Feld durch den Energietensor bedingt und bestimmt sei." (My translation). For discussions on the precise influence of Mach on Einstein, the question of how adequate Einstein's reading of Mach was, and other possible formulations of Mach's principle, see Hoefer [1994], Barbour and Pfister [1995], Renn [2007] and Barbour [2007]. 
of bodies. Since according to the results of the special theory of relativity mass and energy are the same, and since energy is formally described by the symmetric energy tensor $\left(T_{\mu \nu}\right)$, Mach's principle says that the $\left[g_{\mu \nu}\right]$-field is constrained and determined by the energy tensor.

Einstein's formulation of Mach's principle is often taken as indicating his commitment to a Leibnizian/relationalist programme: spacetime was supposed to be secondary to (the mass-energy distribution of) material objects. ${ }^{4}$ Famously, GR does not fulfil Mach's principle as defined above; for example, the original gravitational field equations

$$
R_{\mu \nu}-\frac{1}{2} g_{\mu \nu} R=\kappa_{E} T_{\mu \nu}
$$

allow empty Minkowski spacetime as a solution, among many other matterfree solutions. Even the modified field equations, in which Einstein introduced the cosmological constant $\lambda$ in order for them to accord with Mach's principle, turned out to allow for non-trivial solutions even if $T_{\mu \nu}=0 .{ }^{5}$ Furthermore, the left-hand side of the Einstein equations represents only part of the geometric structure - the Ricci curvature $R_{\mu \nu}$ - whereas the Weyl curvature $C_{\mu \nu \sigma \omega}$ is only constrained but not determined by the energy tensor $T_{\mu \nu} \cdot{ }^{6}$

We will see that even if knowing the energy tensor would uniquely determine the geometric structure, and hence even if Einstein's formulation of Mach's principle was fulfilled, there would still be no reason to regard matter as more fundamental than spacetime in the theory. For the only thing

\footnotetext{
${ }^{4}$ In a footnote, Einstein points out that up to the publication of this article (in 1918), he had not distinguished between what he now called Mach's principle and the relativity principle. In Einstein [1918], p.38, the relativity principle is defined in the following way (which is quite different from his earlier formulations): "The laws of nature are statements about spatio-temporal coincidences; hence they find their only natural expression in generally covariant equations."

${ }^{5}$ See Hoefer [1994] for details.

${ }^{6}$ Earman [1989], p. 107, points out that "the idea that the distribution of mass-energy determines the metric fares even worse in the initial-value problem for Einstein's field equations. [...] Einstein's field equations constrain the initial data through elliptic partialdifferential equations that imply that the initial matter distribution cannot be specified independently of the intrinsic spatial geometry and the extrinsic curvature of the initalvalue hypersurface."
} 
that would really suggest that either matter or geometry was more fundamental would be if the existence of one was a requirement for the existence of the other but not vice versa. We will see that spacetime (and its geometric structure) can exist without matter, whereas systems cannot possess mass-energy-momentum density without spacetime structure being in place. ${ }^{7}$

I will start out in section 1 by arguing that what we mean (or should mean) by 'a material system' when taking into account relativistic field theory is a system which has a mass-energy-momentum tensor $T_{\mu \nu}$ associated with it. In section 3, I shall argue that although most energy tensors depend on the metric tensor explicitly (there is a functional dependence), this is not the crucial kind of dependence of energy tensors on the metric. Section 4 and 5 will then show that the very definition of energy tensors, and the properties they need to have in order to qualify as physical energy tensors, depends on the metric field $g_{\mu \nu}$ in a variety of ways; there could be no energy tensor without the spacetime structure encoded in the metric. Hence, the main property of matter depends on the relations that hold between material systems and spacetime structure; so that section 6 argues that mass-energymomentum is a relational property of matter.

A letter of Einstein to Felix Pirani, written on 2 February 1954, shows how much Einstein had changed his mind on the issue; he writes that ${ }^{8}$

[Proponents of Mach's principle] think that the field should be completely determined by matter. But this is tricky, for the $T_{i k}$, which are supposed to represent "matter", always presuppose the $g_{i k}$ field. [...] [O]ne should not speak of Mach's principle anymore.

Making the second sentence precise is the central purpose of this paper.

\footnotetext{
${ }^{7}$ This result does not in itself decide the substantivalist/relationalist debate. If the relationalist manages to derive the metric field $g_{\mu \nu}$ from the relations between material systems, then my results would be perfectly compatible with a relationalist interpretation of GR. However, the burden of proof is on the relationalist's shoulders; cf. also footnote 33 .

8 "[Machianer] denken, dass das Feld völlig bestimmt sein soll durch die Materie. Dies ist aber eine heikle Sache, da die $T_{i k}$, welche die "Materie" darstellen sollen, immer schon das $g_{i k}$ Feld voraussetzen. [...] Von dem Mach'schen Prinzip aber sollte man nach meiner Ansicht überhaupt nicht mehr sprechen." (My translation.) Cao [1997], p. 80, quotes the first sentence, whereas many other sources, for example Hoefer [1994], p.330, quote the second. For the full letter see Document 17-448 of the Einstein Archive.
} 


\section{The concept of matter in relativistic field theory}

Imagine standing in front of an unremarkable-looking house, spatially extended as houses are. You stretch out your hand to get hold of the doorknoband your hand goes straight through. You feel no resistance, no solidity, and hence have no sense of touch. Would you think of it as a house? Maybe. But would you think of it as a material house? Probably not, you might think of it as a ghost house at best. Then again, imagine a point particle like the electron that has no (so far) measurable extension but which does possess mass. Do you think of it as material? Probably. After all, it can push things.

Back in the 17th century, there were two important opinions about the nature of matter. Descartes claimed that it was the property of being extended that made something material, whereas Newton argued that it was the property of possessing (inertial) mass. ${ }^{9}$ It is the concept of inertial mass $m_{I}$ and momentum $\vec{p}=m_{I} \cdot \vec{v}$, where $\vec{v}$ is the velocity of the body, that gives us an account of the vague intuitions mentioned above: according to Newtonian physics only a body with inertial mass will let us feel resistance when we touch it. For this is the definition of inertial mass: the extent to which a body resists a change of its state of motion. Furthermore, only a body with momentum can push things, has the potential to change the state of motion of other bodies.

Then, at the end of the 19th century, there was a startling development: it was found that electromagnetic fields can push things as well. More precisely, Maxwell, Poynting, Heaviside and J.J. Thomson, building on earlier ideas from W. Thomson and Faraday, found that an electromagnetic field

${ }^{9}$ This is a very simplifying way of putting Newton's position in particular. It is clear that Newton's primary notion of mass is to define it as a measure of matter "that arises from its density and volume jointly" (Definition 1 of the Principia, see Newton [1999]). However, as Biener and Smeenk [forthcoming] argue, one has to distinguish between a geometrical and a dynamical conception of matter, both of which are present in Newton's writings, and each of which corresponds to a different way of measuring mass as defined in Definition 1. In De Gravitatione (see Newton [1962]), presumably written before the first edition of the Principia, Newton defends what Biener and Smeenk call "the geometrical conception of matter", according to which a body's quantity of matter is measured by the volume which the body impenetrably fills. This conception is much closer to Descartes' conception of matter than the one dominant in the Principia, where Newton argues that a body's quantity of matter is measured by its response to impressed force, i.e. by its inertial mass. 
possesses energy and momentum (but no rest mass). After this, physicists came to think of electromagnetic fields as something like matter. Some, most prominently Gustav Mie, even tried to reduce 'ordinary' matter to electromagnetic fields, thinking of the inertial mass possessed by an electron as something to be derived by assuming that it is only a particular configuration of an electromagnetic field. This programme was ultimately unsuccessful, but once it was one of the biggest hopes of theoretical physics. ${ }^{10}$

While the electromagnetic programme was still at its high-point, something surprising happened yet again. The special theory of relativity (SR) was discovered/created, and with it electromagnetic fields, particles and material continua (such as extended bodies and fluids) could be described in a framework that made them look even more like the same kind of thing: all the previously separate properties mentioned above could be encoded by the special-relativistic mass-energy-momentum density tensors. Thus, it became sensible to regard possession of mass-energy-momentum as the property that qualifies a system as a material system above all else.

\subsection{A short history of energy-momentum tensors}

Einstein [1905a] had formulated special relativity and shown, in Einstein [1905b], that an important consequence of it was the famous connection (or 'equivalence') between energy and inertial mass. Minkowski [1908a,b] provided a 4-dimensional reformulation of the theory, showing that the Lorentztransformations are analogous to rotations in 4-dimensional spacetime. As a consequence, coordinates were written in a manner analogous to 4 -vectors, with three space- and one time-component - and with them, all the fundamental quantities of the theory were rewritten in such a way. Most importantly for us, 3-momentum and mass/energy were reconsidered as components of a 4-vector, with mass/energy occupying the 'time-slot' of the vector.

In pre-relativistic physics, it was already well known that a momentum 3 -vector and a scalar quantity describing mass and/or energy, while sufficient for describing point particles, were not sufficient for describing continuum matter. In order to do that, quantities describing energy densities, en-

\footnotetext{
${ }^{10}$ The earliest explicit statement in favour of a reduction of physics to electromagnetism can be found in Wien [1900], but it found its most detailed and promising presentation in the works of Gustav Mie, who built on work of Max Abraham. For summaries and discussion of Mie's programme see Vizgin [1994], pp.26-38, Corry [1999] and Smeenk and Martin [2007].
} 
ergy current densities, momentum densities and momentum current densities within the continuum were needed. In 1905, there existed already a sophisticated non-relativistic theory describing these quantities for the paradigm cases of classical continuum matter - extended bodies with internal stress, fluids, and electromagnetic fields.

Judged from today's perspective, the natural first step to generalise the energy-momentum 4-vector of a free particle might seem to be the search for a quantity describing the energy-momentum properties of a system of many particles - i.e. to develop the relativistic dynamics of stressed bodies and fluids. However, in the early days, special relativity was very closely associated with electrodynamics, and so one of the first tasks Minkowski set himself was to give a 4-dimensional reformulation of relativistic electrodynamics (Minkowski [1908b]).

In doing so, he showed that the energy (current) density, momentum (current) density and stress associated with the electromagnetic field in prerelativistic physics can be seen as components of a second-rank tensor $T_{\mu \nu}$, the mass-stress-energy-momentum density tensor of the electromagnetic field. ${ }^{11}$ It was mostly through the work of Max von Laue that Minkowski's way of constructing an energy tensor was extended from electromagnetism to 'ordinary matter' such as extended stressed bodies, material dust, and relativistic fluids (von Laue $[1911 b, a]$ ). ${ }^{12}$ Just as Minkowski had done for electromagnetic fields, von Laue showed that the familiar quantities describing e.g. stress and energy of an extended body or a fluid could be regarded as components of a second-rank tensor $T_{\mu \nu}$ associated with the material system, while including the relativistic equivalence between mass and energy in the $T_{00}$ component of this tensor. Recently, Ohanion [2008] has argued that all of Einstein's proofs of mass-energy equivalence fail for extended material systems whose internal motions are relativistic, and that von Laue should be given credit for having proven mass-energy equivalence for general material systems with arbitrary mass-energy-momentum tensor $T_{\mu \nu}$. One might call energy-momentum tensors obtained in the above (constructive) manner Laue energy tensors, to be contrasted with the (deductively obtained) Hilbert energy tensors described below.

The following paragraph from one of Einstein's unpublished manuscripts

\footnotetext{
${ }^{11}$ Again, I shall often speak simply of the 'energy-momentum tensor' or even just of the 'energy tensor' of a material system.

${ }^{12}$ See also Norton [1992], especially pp.28-33 for a summary of von Laue's development of a general energy-momentum tensor for stressed bodies and fluids.
} 
(written in 1912) makes evident the importance Einstein attributed to the development of energy-momentum tensors: ${ }^{13}$

The general validity of the conservation laws and of the law of the inertia of energy $[\ldots]$ suggest that [the symmetric energymomentum tensor $T_{\mu \nu}$ of a given system and the equation $K_{\mu}=$ $\partial^{\nu} T_{\mu \nu}$ where $K_{\mu}$ is the external 4-force acting on the system] are to be ascribed a general significance, even though they were obtained in a very special case [i.e. electrodynamics]. We owe this generalisation, which is the most important new advance in the theory of relativity, to the investigations of Minkowski, Abraham, Planck, and Laue. [...] To every kind of material process we want to study, we have to assign a symmetric tensor $\left(T_{\mu \nu}\right) \ldots$.

Thus we see that soon after special relativity was formulated it was claimed that every kind of matter has an energy tensor associated with it, encoding the new relativistic concepts of mass, energy, momentum and stress. The difference between electromagnetic fields and 'ordinarily material' continua such as fluids and extended bodies was reduced to their having energy tensors of different form: for electromagnetic fields, the sum of the diagonal components (the trace) $T_{\mu}^{\mu}$ of the energy-momentum tensor necessarily vanishes (in every coordinate system), for ordinary continua its trace is necessarily non-zero. ${ }^{14}$ Hence, it now seemed justified to think of electromagnetic fields

\footnotetext{
13 "Die Allgemeingültigkeit der Erhaltungssätze und des ... Satzes von der Trägheit der Energie legen es nahe, [dem symmetrischen Energie-Impuls Tensor $T_{\mu \nu}$ eines gegebenen Systems und der Gleichung $K_{\mu}=\partial^{\nu} T_{\mu \nu}$, wobei $K_{\mu}$ die auf das System wirkende externe 4-Kraft ist,] obwohl sie an einem ganz speziellen Falle gefunden sind, eine allgemeine Bedeutung zuzuschreiben. Wir verdanken diese Verallgemeinerung, welche den wichtigsten neueren Fortschritt der Relativitätstheorie bildet, den Untersuchungen von Abraham, Minkowski und Laue. Wir haben jeder Art studierter materieller Vorgänge einen symmetrischen Tensor $\left(T_{\mu \nu}\right)$ zuzuordnen... ." See the Collected papers of Albert Einstein, Vol. 4, Document 1 (p. 92); the emphasis is mine (apart from 'general'), whereas the translation is from the corresponding English volume of the CPAE. Cf. also Janssen and Mecklenburg [2007], pp. 41-50, who elaborate how the transition "from the electromagnetic view of nature to relativistic continuum mechanics" was performed.

${ }^{14}$ This mathematical difference encodes an important physical difference between relativistic fluids and extended bodies on the one hand and electromagnetic fields on the other: in describing the former it is always possible to go to a local rest frame of a given volume element of the fluid, in which $T_{\mu \nu}$ turns out to have only diagonal components, whereas for electromagnetic fields no such rest frame is defined; light never rests. See Landau and Lifschitz [1966], p.505 and Landau and Lifschitz [1989], p.82 and p.92, for details.
} 
as really a kind of matter.

When Einstein started to search for a relativistic theory of the gravitational field, it seemed natural to expect the newly found energy tensor to play the role of the 'source' of gravitational fields in much the same way as the mass density $\rho_{m}$ played this role in Newtonian gravitation theory, and as the electric charge density $\rho_{e}$ does in electrostatics. Hence in the context of searching for gravitational field equations, Einstein and Grossmann in their 1914 Entwurf theory put constructed energy tensors on the right-hand side of their candidate field equations, even after having derived the vacuum field equations from a Lagrangian approach (Einstein and Grossmann [1914]; Einstein [1914], especially pp. $97-109$ of the former). ${ }^{15}$

Hilbert, on the other hand, assumed that the total Lagrangian depends not only on the metric field $g_{\mu \nu}$ (corresponding, for both him and Einstein, to the gravitational potential), but also on the electromagnetic potential $A_{\mu}$. Thus he postulated that this Lagrangian would ultimately describe both matter and gravitational fields. Hilbert was licensed to do this because he followed Mie's programme, which hypothesised that all matter could eventually be reduced to electromagnetic fields. Hilbert was criticised for this speculative assumption by Einstein [1916] and Weyl [1917], who rederived the energy tensor in a way similar to Hilbert, but without assuming that the fundamental matter fields were only electromagnetic in nature. They allowed matter fields to be of arbitrary rank and to possess symmetry properties different from those of the antisymmetric electromagnetic field tensor $F_{\mu \nu}$. They thereby introduced the modern concept of a general matter field $\Phi$. It seems to be the pattern that, since then, introducing a new field into the inventory of physics is spoken of as introducing a new matter field if the new field has a mass-energy-momentum tensor associated with it. ${ }^{16}$

\footnotetext{
${ }^{15}$ Even though it is natural to expect $T_{\mu \nu}$ to play the role of the gravitational field source, it is not a priori so. Indeed, there once was a candidate for the correct relativistic theory of gravitation which did not have the full energy tensor on the right-hand side of the field equation, but only its trace $T_{\mu}^{\mu}$ : Nordström's theory of gravitation, developed between 1912 and 1914. (For a historical review of Nordström's theory and the exchange between Einstein and Nordström see Norton [1992], for a systematic discussion of the possibility of relativistic scalar gravitation theories see Giulini [2008].) In section 4, I shall argue that $T_{\mu \nu}$ is not a source in quite the same way as $\rho_{m}$ and $\rho_{e}$ after all.

${ }^{16}$ Note that although Hilbert's assumption of the electromagnetic nature of matter is questionable as an assumption of principle, the restriction to the case where only electromagnetic fields are present in addition to gravitational ones became a field of intense study, and Hilbert's form of the field equations became known as the Einstein-Maxwell
} 
So whereas Einstein and Grossmann originally took their energy tensors 'off the shelf', Hilbert derived the energy tensor of the electromagnetic field from a Lagrangian that also gave the free Einstein and the free Maxwell equations. Hilbert saw this result as "the triumph of axiomatics" (cf. Kichenassamy [1993], p. 196). The result seemed particularly remarkable because Hilbert, being a follower of Mie's programme, thought that only the energy tensor of the electromagnetic field was fundamental, i.e. he thought of von Laue's energy tensors describing fluids and extended bodies as merely effective, ultimately to be derived from the behaviour of electromagnetic fields. ${ }^{17}$

Even though this hope was not fulfilled, it was subsequently found that the energy tensors of all paradigmatic material systems formalisable in a Lagrangian framework can be derived in a similar manner: which seems remarkable enough. I will call energy tensors obtained (or even defined) in such a way Hilbert energy tensors, ${ }^{18}$ and will review a modern version of the derivation scheme in section $4 .{ }^{19}$

equations. Furthermore, some of Einstein's work on unified field theories in the 1920s can be read as involving Einstein making assumptions very similar to Hilbert's. Indeed, Hilbert saw Einstein's work on affine field theory as "a collosal detour via Levi-Civita, Weyl, Schouten, Eddington [...]" back to Hilbert's 1915 theory, cf. Sauer and Majer [2005]. However, Einstein always expected electromagnetic fields and gravitational fields together to constitute (apparently) particle-like matter.

${ }^{17}$ See Sauer [1999], especially p.555, and Brading and Ryckman [2008] for more details on this aspect of Hilbert's work.

${ }^{18}$ This terminology pops up in certain texts when referring to the energy tensors derived with the help of Lagrangians, but it is not a universally used terminology.

${ }^{19} \mathrm{~A}$ more detailed account of the history of energy-momentum tensors would now go on to describe the Noether theorems and currents, giving rise to the definition of so-called canonical energy tensors in special relativity. It would report in detail the discussion of whether canonical energy tensors and Hilbert energy tensors are after all equivalent, even though canonical energy tensors (in contrast to Hilbert tensors) are not necessarily symmetric but have to be symmetrised by a procedure first found by Belinfante [1940] and Rosenfeld [1940]. Very recently, Leclerc [2006] proposed a proof to show that canonical energy tensors and Hilbert tensors are indeed equivalent if matter fields do not couple to the derivatives of the metric field, while Saraví [2002] explicitly showed the equivalence between the canonical and the Hilbert energy tensor for the free electromagnetic field. Another important topic on the purely mathematical side is Petrov's and Serge's classification of second-rank tensors, classifying both the Ricci and energy tensors in a rigourously algebraic manner: see Hall [2004]. 


\subsection{Metaphysical matters}

We started out by saying that we think of something as material if we feel resistance when trying to touch it, and if we know that it could push other things given certain circumstances, i.e. if it has inertial mass and hence the potential to possess energy and momentum. Then we found that all these concepts have been unified and generalised in relativity theory by the introduction of mass-energy-momentum density tensors $T_{\mu \nu}$, encoding these properties for particles, extended bodies, fluids, electromagnetic fields - basically everything we could think of as material. It seems that intuitively we think of something as matter only if there is mass, energy and/or momentum associated with it, and if we find these properties associated with something which we did not think of as matter beforehand, we will now do so (remember how opinion changed about the 'materiality' of the electromagnetic field). In other words: it seems we regard possession of mass-energy-momentum as an essential, rather than as an accidental, property for something to count as material.

The distinction can be introduced in the following way: An essential property of an object is a property that it must have, if it exists, while an accidental property of an object is one that it happens to have but that it could lack, even if it exists. In our case this means that if something is material then it must have mass-energy-momentum. An object might lack the property $T_{\mu \nu}$ and still exist - but it cannot lack $T_{\mu \nu}$ and still count as a material object. ${ }^{20}$

Note that an essential property does not need to be a fundamental property, i.e. a property that cannot be defined in terms of other properties. Indeed, we will now see that, despite the above, mass-energy-momentum density is not a fundamental property in relativistic field theory.

\section{Explicit dependence of energy tensors on the metric field}

The fundamental mathematical objects in relativistic field theory are tensor fields like $F_{\mu \nu}, \phi$ and $g_{\mu \nu}$. We often speak of $F_{\mu \nu}$ as 'an electromagnetic field', of $\phi$ as 'a scalar field' and of $g_{\mu \nu}$ as 'a metric field', and indeed one can

\footnotetext{
${ }^{20}$ See Robertson [2008] for the above definition of essentiality.
} 
interpret the tensor fields as referring to the physical fields in a rather direct way. But one can also argue that $F_{\mu \nu}$ does not represent the electromagnetic field 'as such', but a certain property, set of properties or even trope of the physical electromagnetic field: its amplitude, or electromagnetic field strength. Nothing I am going to say depends on this choice; although I favour the interpretation that regards $F_{\mu \nu}$ as representing the fundamental properties of the material system described by $F_{\mu \nu}$.

If one endorses this interpretation, one should note the following (and corresponding facts if one makes a different interpretational choice): the fundamental properties of a material system do not need to be describable by only one tensor field. The fundamental properties of a perfect fluid for example are described by a triple of matter fields, $\left(\rho, v^{\mu}, p\right)$, where $\rho$ is the proper density of the particles the fluid consists of, $v^{\mu}$ the velocity field describing the movement of every particle, and $p$ the pressure field, giving the force an arbitrary fluid volume element 'feels' due to the movement of the rest of the fluid.

I follow the common custom and call a tensor field $\Phi$ a 'matter field' if it describes a material system. Indeed, I have argued above that what makes us think of systems being described by $F_{\mu \nu}$ or $\left(\rho, v^{\mu}, p\right)$ as material systems is that they possess mass-energy-momentum (density). But whereas mass density was represented by a simple scalar field in Newtonian physics, relativistic mass-energy-momentum density $T_{\mu \nu}$ is defined in terms of the fundamental matter fields associated with the material system. But this is not enough: energy tensors also depend on the metric field $g_{\mu \nu}$ !

One could think that this is obvious, for in their coordinate-independent representation the energy tensors of almost all paradigm material systems contain the metric field explicitly.

For example, the energy tensor of an electromagnetic field is

$$
T_{\mu \nu}=\frac{1}{4 \pi}\left(F_{\mu}{ }^{\lambda} F_{\lambda \nu}+\frac{1}{4} g_{\mu \nu} F^{\sigma \lambda} F_{\sigma \lambda}\right),
$$

while the energy tensor for a perfect fluid is given by

$$
T_{\mu \nu}=(\rho+p) v_{\mu} v_{\nu}-p g_{\mu \nu} .
$$

However, this functional dependence on the metric does not tell us much. First of all, it is not a feature that all energy tensors share: there are two particularly simple systems whose energy tensor does not depend on the 
metric explicitly. One of them is a specialisation of a perfect fluid, namely a perfect fluid without pressure, also called dust for short. The energy tensor of this system is

$$
T_{\mu \nu}=\rho v_{\mu} v_{\nu} .
$$

One might think that a material system whose energy tensor depends on the metric explicitly might be 'more' dependent on the metric field $g_{\mu \nu}$, and in particular more sensitive to changes in the metric from point to point. But this is not the case. For if a metric is defined we can write equation (4) equally well as

$$
T_{\mu \nu}=\rho g_{\alpha \mu} g_{\beta \nu} v^{\alpha} v^{\beta},
$$

and it will still represent the same physics. In particular, curvature will not influence a system represented by equation (5) any more than a system represented by equation (4). And of course the important consideration is not whether something can be written with $g_{\mu \nu}$ in it, but whether it cannot be written without it.

The next two sections will show that the relevant dependence of energy tensors on the metric field is not a functional dependence but a definitional one. Indeed, we shall see that the definition of energy tensors depends on the metric tensor field in a variety of ways.

I shall first show how energy tensors can be derived in a Lagrangian framework, whereby it will become clear that in order for a material system to possess mass-energy-momentum in this framework, a certain relation must obtain between the matter fields and the metric field, namely that the matter fields and the metric field couple in a certain way.

\section{Metric dependence in Lagrangian theories}

\subsection{The basic ideas of Lagrangian field theory}

The basic idea of Lagrangian field theory is to derive the equations of motion (also called the field equations) of a field $\Psi$ by using the calculus of variations. One starts by assigning to the fields a Lagrangian density $\mathscr{L}$, or Lagrangian for short, with the help of which the action $S$ can be defined: ${ }^{21}$

\footnotetext{
${ }^{21}$ Some authors reserve 'Lagrangian' for the integral of the Lagrangian density over a spatial hypersurface. Note that strictly speaking $\mathscr{L}$ in equation (6) also depends on the
} 


$$
S=\int_{\Omega} \mathscr{L}\left(\Psi, \partial_{\mu} \Psi, \partial_{\nu} \partial_{\mu} \Psi, \ldots\right) d \Omega
$$

$\Omega$ is a compact region in spacetime, $d \Omega$ a 4 -dimensional volume element. The field $\Psi$ can be a tensor field of arbitrary rank. The dots in the Lagrangian signify that in principle it could depend on (partial or covariant) derivatives of the fields of arbitrary (finite) order. However, it is almost always enough to allow the Lagrangian to depend only on the first and second derivatives of the fields $\Psi$ in order to derive their equations of motion. ${ }^{22}$

In general relativistic systems, there are two kinds of fields: the matter fields $\Phi$ and the metric/gravitational field $g_{\mu \nu}$. We shall see that the matter fields can only represent material systems if there is a metric field as wellthat it is only because of their relation to the metric field that they have a mass-energy-momentum tensor $T_{\mu \nu}$ associated with them.

In any case, the idea is that the Lagrangian is a scalar functional (up to a total divergence) of the dynamical fields present in the system under consideration, and that if one knows the Lagrangian, one can deduce everything else by varying the fields and imposing certain constraints on the variations. The variation of a field $\Psi$ is given by $\Psi \rightarrow \Psi+\delta \Psi .^{23}$

coordinates $x^{\mu}$, but it is common to omit writing out this dependence explicitly.

${ }^{22}$ There are many textbooks on Lagrangian field theory; a good place to start is Hobson et al. [2006], who deliver a very concise treatment in the context of GR and make particularly clear the differences and similarities between matter fields and metric field. Furthermore, classics like Landau and Lifschitz [1989], Hawking and Ellis [1973], and Wald [1984] give excellent treatments of the role variational methods play in GR, whereas Lanczos [1986] delivers a wonderful systematic-historic discussion of variational principles in general. Poisson [2004] gives a detailed presentation of the boundary terms which have been found necessary in order to make the variational problem for the case of the Hilbert Lagrangian (16) well-defined.

${ }^{23} \mathrm{~A}$ more abstract way of defining the notion of a variation is found by considering a smooth one-parameter family of fields $\Psi_{\lambda}$ which start from $\Psi_{0}$ and satisfy appropriate boundary conditions. A variation is then defined by the equation $\delta \Psi:=\left.\frac{d \Psi_{\lambda}}{d \lambda}\right|_{\lambda=0}$. For this way of defining variations see e.g. Wald [1984], p.450, and Hawking and Ellis [1973], p.65. For the definition of the variation used in the main text see for example Hobson et al. [2006], p.87 and 529. For a proof that variation $\delta$ and integration $\int$ commute see Hobson et al. [2006], p.88, while commutation between variation and partial/covariant derivatives is proven on p.529 and p.531. Note also that Kichenassamy [1993] distinguishes three different kinds of variation, which he terms functional variation, label variation and Lie variation, and suggests that Hilbert [1915] and Einstein [1916] did not clearly distinguish between them. Label variation and Lie variation are essential in proving conservation laws; in this text, we shall need only what Kichenassamy calls the functional variation. 
We now impose Hamilton's principle for the variation of the matter fields, namely that the variation $\delta S$ of the action be stationary under variations of the fields. More precisely, we demand that $\delta S=0$ for arbitrary variations $\delta \Psi$, if the variation vanishes on the boundary $\partial \Omega$ of the volume element $d \Omega$. It can be shown that a necessary and sufficient condition for the action to be stationary is the fulfilment of the so-called Euler-Lagrange equations of the system, which are equivalent to the equations of motion of the fields given a suitable Lagrangian $\mathscr{L} .{ }^{24}$ For an arbitrary matter field $\Phi$, whose Lagrangian depends only on the field and its first order covariant derivatives (and the metric, as we will see further below), the Euler-Lagrange equations are

$$
\frac{\delta \mathscr{L}}{\delta \Phi}:=\frac{\partial \mathscr{L}}{\partial \Phi}-\nabla_{\mu}\left(\frac{\partial \mathscr{L}}{\partial\left(\nabla_{\mu} \Phi\right)}\right)=0
$$

where $\frac{\delta \mathscr{L}}{\delta \Phi}$ is called the variational derivative, or Euler-Lagrange derivative, of $\mathscr{L}$ with respect to $\Phi$.

\subsection{Enter the energy tensor}

In the above, we have seen that varying the matter fields and imposing Hamilton's principle gives us the equations of motion of the matter fields. But the Lagrangians for material systems depend not only on the matter fields $\Phi$ and their derivatives but also on the metric field $g_{\mu \nu}$ ! (The Lagrangian for the metric field, on the other hand, does not depend on any other fields.)

Somewhat surprisingly, it is only via the dependence of matter Lagrangians on the metric field $g_{\mu \nu}$ that we can derive what makes us think of them as matter fields: an associated mass-energy-momentum tensor $T_{\mu \nu}$. For example, the variational derivative of the electromagnetic Lagrangian ${ }^{25}$

$$
\mathscr{L}_{E M}\left(A_{\mu}, \nabla_{\nu} A_{\mu}, g_{\mu \nu}\right)=\frac{\sqrt{-g}}{8 \pi} g^{\alpha \gamma} g^{\beta \delta} F_{\alpha \beta} F_{\gamma \delta}
$$

with respect to the metric field, $\frac{\delta \mathscr{L}_{E M}}{\delta g_{\mu \nu}}$, gives us a generalised version of

\footnotetext{
${ }^{24}$ For a proof that the fulfilment of the Euler-Lagrange equations is a necessary and sufficient condition for the action to be stationary see for example Hawking and Ellis [1973], p. 65; and cf. Doughty [1990], who on p. 178 points out that this is the case only if the Euler-Lagrange equations are consistent.

${ }^{25}$ The Faraday tensor $F_{\mu \nu}$ is defined in terms of the electromagnetic vector potential $A_{\mu}$ by $F_{\mu \nu}=\nabla_{\nu} A_{\mu}-\nabla_{\mu} A_{\nu}=\partial_{\nu} A_{\mu}-\partial_{\mu} A_{\nu}$.
} 
Minkowski's energy tensor of the electromagnetic field: ${ }^{26}$

$$
T_{\mu \nu}=\frac{1}{4 \pi}\left(F_{\mu}{ }^{\lambda} F_{\lambda \nu}+\frac{1}{4} g_{\mu \nu} F^{\sigma \lambda} F_{\sigma \lambda}\right)
$$

The first thing to note is that a similar procedure is available for every matter field $\Phi$ : given a Lagrangian $\mathscr{L}_{\Phi}$ that gives us the equations of motion for $\Phi$, the same Lagrangian will give us the energy tensor of the field $\Phi$, when the variational derivative with respect to the metric field $g_{\mu \nu}$ is taken. The important difference is that in order to obtain the Euler-Lagrange equations for $\Phi$, we impose Hamilton's principle on variations of $\Phi$, while we do not do so when calculating $\Phi$ 's energy tensor from variations of $g_{\mu \nu}$ (I shall comment more on this at the end of the section). This procedure turns out to be so successful in recovering energy tensors previously constructed by phenomenological means that in mathematical physics

$$
T_{\mu \nu}:=-\frac{2}{\sqrt{-g}} \frac{\delta \mathscr{L}_{\Phi}\left(g_{\mu \nu}, \Phi, \nabla_{\mu} \Phi\right)}{\delta g^{\mu \nu}}
$$

is often used as the definition of the energy tensor of a newly introduced matter field $\Phi$.

Note that even if the matter Lagrangian depends on more than one matter field, we never have a term in the total matter Lagrangian that depends only on a matter field but not on the metric field. ${ }^{27}$ Hence, it follows from equation (10) that the energy tensor of a material system vanishes if and only if the corresponding matter field(s) $\Phi$ vanishes. For the latter tells us that $T_{\mu \nu}$ can vanish in a certain region only if $\Phi$ vanishes in the same region (for $g_{\mu \nu}$ never vanishes), and it tells us that if $T_{\mu \nu}$ vanishes then $\Phi$ must vanish also. As a consequence, every non-vanishing matter field $\Phi$ brings with it a non-vanishing energy tensor $T_{\mu \nu}$ and vice versa. ${ }^{28}$ Hence, the presence of a non-vanishing energy tensor $T_{\mu \nu}$ at a point is a necessary and sufficient condition for this point to be occupied by matter.

\footnotetext{
${ }^{26}$ It is a generalised version because Minkowski formulated his energy tensor for special relativistic systems, whereas this energy tensor, allowing for a dynamical metric, holds in general relativity as well.

${ }^{27}$ Brown and Brading [2002] suggest that general covariance (and the role it plays in conservation laws as obtained from Noether's theorems) rules out the possibility of having a Lagrangian that depends only on the matter fields but not on the metric field. See in particular p.3 and section $\mathrm{V}$ of their paper.

${ }^{28}$ Cf. Hawking and Ellis [1973], p.61.
} 
Nevertheless, it is only via the dependence of the matter Lagrangian on both the matter field $\Phi$ and the metric field $g_{\mu \nu}$ that we can define an energy tensor for the material system represented by $\Phi$.

Our task is now to make more precise the kind of dependence on $g_{\mu \nu}$ that a matter Lagrangian must exhibit in order for it to be possible to define an energy tensor for the respective material systems. In order to do this, we need to distinguish between the different kinds of coupling of fields that are possible in Lagrangian field theories.

\subsection{Different kinds of coupling}

Imagine that there are only two non-gravitational fields in the world, say a scalar field $\phi$ and an electromagnetic field $F_{\mu \nu}$, with Lagrangians

$$
\mathscr{L}_{\phi}=\frac{\sqrt{-g}}{2} g^{\mu \nu} \nabla_{\mu} \phi \nabla_{\nu} \phi
$$

and

$$
\mathscr{L}_{E M}=\frac{\sqrt{-g}}{8 \pi} g^{\alpha \gamma} g^{\beta \delta} F_{\alpha \beta} F_{\gamma \delta}
$$

respectively. Then there is a clear distinction between the case where the two fields are interacting directly and the case where they are not interacting directly. What is more, both cases are physically possible! For the total Lagrangian of the physical system could either be

$$
\mathscr{L}_{1}=\mathscr{L}_{\phi}+\mathscr{L}_{E M}
$$

or

$$
\mathscr{L}_{2}=\mathscr{L}_{\phi}+\mathscr{L}_{E M}+\mathscr{L}_{\text {int }}
$$

where $\mathscr{L}_{\text {int }}$ describes the direct interaction between the two fields. For example, it could be

$$
\mathscr{L}_{\text {int }}=\sqrt{-g} \omega^{2} \phi F_{\mu \nu} F^{\mu \nu}
$$

where $\omega$ is the coupling constant. In this case, $\phi$ and $F_{\mu \nu}$ (or $A_{\mu}$, in terms of which $F_{\mu \nu}$ is defined) directly and minimally couple to each other.

Two fields are said to directly couple if they are factors of the same product term in the Lagrangian, which then gives coupled differential equations as 
field equations via the Euler-Lagrange equations. They are said to indirectly couple if they do not couple directly but via an intermediate field: if field $\phi$ directly couples to field $g_{\mu \nu}$ and field $g_{\mu \nu}$ directly couples to field $F_{\mu \nu}$, then field $\phi$ indirectly couples to field $F_{\mu \nu}$. Another example is Lagrangian (13): the scalar field $\phi$ and the electromagnetic field $F_{\mu \nu}$ indirectly couple to each other via the metric field $g_{\mu \nu}{ }^{29}$

A field $\phi$ is said to minimally couple to a field $F_{\mu \nu}$ if it couples only to $F_{\mu \nu}$ and/or its (possibly covariant) derivatives; but not to a tensor formed from the field and its derivatives. In particular, minimal coupling of a matter field $\Phi$ to the metric field $g_{\mu \nu}$ means that there is no coupling to the curvature tensor $R_{\mu \nu \sigma}^{\omega}$, which is definable in terms of the partial derivatives of the metric field. Minimal coupling can happen either directly or indirectly. ${ }^{30}$

Now imagine a world in which we have only an electromagnetic field $F_{\mu \nu}$ and a metric field $g_{\mu \nu}$. For the latter, the field equations (the Einstein equations) in the absence of matter are obtained from the Hilbert Lagrangian

$$
\mathscr{L}_{G}=\sqrt{-g} R
$$

where $R$ is the Ricci scalar.

In the presence of an electromagnetic field, it is not possible to write the total Lagrangian in a form corresponding to equation (14), i.e.

$$
\mathscr{L}_{\text {tot }}=\mathscr{L}_{G}+\mathscr{L}_{E M}+\mathscr{L}_{\text {int }},
$$

where only $\mathscr{L}_{\text {int }}$ would contain both fields. For, as we have seen in the last subsection, $\mathscr{L}_{E M}$ already contains the metric field $g_{\mu \nu}$, and in order for the electromagnetic field to have mass-energy-momentum, $\mathscr{L}_{E M}$ must indeed depend on $g_{\mu \nu}$. And so $\mathscr{L}_{E M}$ exactly fulfils our definition of what it means for one field to directly and minimally couple to another field: $g_{\mu \nu}$ and $F_{\mu \nu}$ so couple in $\mathscr{L}_{E M}$. We hence see that what would normally be the interaction

\footnotetext{
${ }^{29}$ Yet another example of indirect coupling is Brans-Dicke theory in the Jordan frame (cf. Weinstein [1996]): the newly introduced scalar field $\phi$ indirectly couples to all matter fields via directly coupling to the metric $g_{\mu \nu}$; which in turn directly couples to all matter fields.

${ }^{30}$ The definition of minimal coupling to the metric via the absence of coupling to the curvature tensor can be found in Goenner [1984]. He argues that the common definition of minimal coupling, namely that general relativistic field equations can be obtained from special relativistic ones by demanding that $\eta_{\mu \nu} \rightarrow g_{\mu \nu}$ and $\partial_{\mu} \rightarrow \nabla_{\mu}$, where $\eta_{\mu \nu}$ is the Minkowski metric and $\nabla_{\mu}$ compatible to $g_{\mu \nu}$, is unique only for first-order differential equations, and hence not generally applicable.
} 
term between metric and electromagnetic field, $\mathscr{L}_{\text {int }}\left(g_{\mu \nu}, F_{\mu \nu}\right)$, is already and necessarily part of the 'free' electromagnetic Lagrangian $\mathscr{L}_{E M}$.

However, note that only direct coupling of a matter field $\Phi$ is a necessary condition to define an energy tensor for this field. For imagine the matter Lagrangian were $\mathscr{L}_{M}=\mathscr{L}_{M_{1}}\left(g_{\mu \nu}, F_{\mu \nu}\right)+\mathscr{L}_{M_{2}}\left(\phi, F_{\mu \nu}\right)$, i.e. we have direct coupling of the matter fields $\phi$ and $F_{\mu \nu}$ to each other, direct coupling of $F_{\mu \nu}$ to the metric field $g_{\mu \nu}$, but only indirect coupling of $\phi$ to the metric. In this case the variational derivative of $\mathscr{L}_{M}$ with respect to $g_{\mu \nu}$ gives the same energy tensor as if $\mathscr{L}_{M}$ had been identical to $\mathscr{L}_{M_{1}}\left(g_{\mu \nu}, F_{\mu \nu}\right)$. In other words, we would get the energy tensor of only the electromagnetic field, for the lack of direct coupling between $g_{\mu \nu}$ and $\phi$ stops $\phi$ from having massenergy-momentum. Thus, direct coupling between the metric field and the fields representing a material system results in the latter having an energy tensor! ${ }^{31}$

The above should not be misunderstood as saying that energy tensors result from an interaction between the matter fields $\Phi$ and the metric field $g_{\mu \nu}$. For an interaction demands that all fields present are dynamical fields, and so such a statement would cause difficulties set against the reality that we can define energy tensors in special relativity (with its non-dynamical metric) equally well as in general relativity (with its dynamical metric). And even in special relativity we can obtain the energy tensor as the variational derivative of the matter Lagrangian, $T_{\mu \nu}=\frac{1}{\sqrt{-g}} \frac{\delta \mathscr{L}_{\Phi}\left(g_{\mu \nu}, \Phi, \nabla_{\mu} \Phi\right)}{\delta g_{\mu \nu}}$; for the definition of the variation of $g_{\mu \nu}$ and the resulting variational derivative with respect to the metric does not depend on the metric field being dynamical or non-dynamical. ${ }^{32}$ Furthermore, even though we can vary $g_{\mu \nu}$, we may not demand that Hamilton's principle holds for the variations of the metric field of special relativity: for otherwise it would be a dynamical field. This is a fortunate prohibition, for if we were allowed to demand $\delta S=0$ for all $\delta g_{\mu \nu}$ in special relativity, it would give us $T_{\mu \nu}=0$ as a consequence, precisely because there is no purely metric Lagrangian in SR. And of course we know that we can (and normally do) have non-vanishing energy tensors even

\footnotetext{
${ }^{31}$ Non-minimal but direct coupling of a matter field to the metric field, on the other hand, would give the energy-momentum tensor (just as the equations of motion) a more complicated structure, but we could still define it. For the (hypothetical) energy-momentum tensor of an ideal fluid whose matter fields couple non-minimally to the metric field but which nevertheless has the special-relativistic energy-momentum tensor of an ideal fluid as a limiting case see Goenner [1996], p.267, equation (9.2).

${ }^{32}$ See Landau and Lifschitz [1989], p.293, for a similar statement.
} 
in special relativistic systems. So even though the matter fields $\Phi$ and the metric field $g_{\mu \nu}$ are interacting in GR, they do not need to interact in order for there to be a non-vanishing energy tensor. What we do need in order to have a non-vanishing energy tensor for every matter field is a weaker requirement: direct coupling of every matter field to the metric field in the matter Lagrangian.

Indeed, it seems sensible to make a distinction between speaking of two fields interacting and two fields coupling. For a dynamical field can couple to a non-dynamical field in the way defined above, but we would not speak of an interaction if only one of the two fields was dynamical: a non-dynamical field acts without being acted upon if it couples to a dynamical field. Hence, two fields interacting should be seen as sufficient but not necessary for the fields to couple, whereas two fields coupling is necessary but not sufficient for the two fields to interact. ${ }^{33}$

To sum up, the matter fields $\Phi$ stand in a certain relation to the metric field $g_{\mu \nu}$, they couple directly to $g_{\mu \nu}$, a relation necessary for us to be able to define mass-energy-momentum $T_{\mu \nu}$ in a Lagrangian framework.

\section{Metric dependence in general}

The last section has discussed how mass-energy-momentum density tensors can be derived in a Lagrangian framework, and that indeed this is only

\footnotetext{
33 In the 'constructive' approach towards special relativity, it has been argued that the metric should not be seen as an entity in its own right, but as merely encoding certain properties of the matter fields; see Brown [2005] and Brown and Pooley [2004] for advocacy, and Norton [2008] and Janssen [2007] for criticism of this position. An argument in support is that an unsatisfactory element of special relativity is believed to be that the metric seems to violate the action-reaction principle by acting without being acted upon: a feature we get rid of if the metric is conceived as encoding properties of the fully interacting matter fields. What I say above does not help to decide for or against a constructive approach, especially since constructivists and anti-constructivists will see it in very different lights. The constructivist will see the dependence of energy tensors on the metric tensor as yet another example of the fundamental difference between special and general relativity: he will say that in SR we have energy tensors depending on something that can be reduced to the properties of the matter fields, whereas with respect to GR he will say that the energy tensor of a given matter field depends on its relation to a new dynamical field, the metric field. The anti-constructivist, on the other hand, will stick with his more unified picture and say that in both GR and SR energy tensors depend on the structure of spacetime, be it dynamical or not.
} 
possible if the matter fields couple directly to the metric field: there is a definitional dependence of energy tensors on the metric tensor in Lagrangian theories. We have thereby made a case to the effect that there is a particular relation between spacetime structure and matter, one that allows the latter to possess mass-energy-momentum. But not every system can be given a Lagrangian formulation - or at least not every system has yet been given one. This is particularly true for complex fluid systems. ${ }^{34}$ In this section, we shall see that even independently of a Lagrangian formulation, we have a definitional dependence of energy tensors on the metric tensor - indeed we have more than one such dependence on the metric. ${ }^{35}$

It should be noted that only some of the ways in which the energy tensor of a particular material system depends on the metric field will apply to all kinds of material systems. It might be suggested that only these latter, most general kinds of dependence should be looked at in detail, and that they sustain the burden of the argument of this paper: that the property of mass-energy-momentum density possessed by material systems depends on their relation to spacetime structure, and metric structure in particular. However, it seems instructive to try to give as complete a picture as possible of the role spacetime needs to play in giving an account of the properties of material systems, especially if there is a kind of dependence on spacetime structure that applies only to some, and another that applies to all material systems. It seems a necessary precaution to distinguish clearly, and it turns out that the most general kinds of dependence on metric structure become particularly clear when compared to the more special cases.

Caution also demands that we distinguish clearly between the possibility of metric structure being sufficient for an energy tensor to be defined, and the possibility of it being necessary to have a metric. Indeed, one might expect that for the definition of the energy tensor of some, particularly simple, material systems, it is enough for spacetime to be endowed with a conformal

\footnotetext{
${ }^{34}$ Hawking and Ellis [1973], pp.69-71, give a Lagrangian description of an isentropic perfect fluid, DeFelice and Clarke [1990], pp.195-8, even give one for a general perfect fluid, and Narlikar [1993], pp.53-7 provides a (hand-waving) Lagrangian description of a perfect fluid without pressure (i.e. of dust, see below). But more complex fluid systems, such as ones involving viscosity and heat transfer, have not to my knowledge been given a Lagrangian formulation.

${ }^{35}$ This section rests on discussions with Robert Geroch, Erik Curiel, Stephen Lyle, John Norton and David Malament, to whom I am indebted for their patient efforts. Needless to say, any unclarities or misconceptions that remain are mine.
} 
rather than a metric structure: where metric structure means that spacetime is endowed with a unique metric tensor field $g_{\mu \nu}$, conformal structure demands only that it is endowed with an equivalence class $\left[g_{\mu \nu}\right]$ of such metrics. ${ }^{36}$

In the case where this class is given by metrics with Lorentzian signature, conformal structure amounts to a light cone being assigned to every point of spacetime. This allows us to distinguish between light-like curves (null curves, forming the boundary of the cone), time-like curves (curves in the interior of the cone) and space-like curves (curves in the exterior of the cone). Metric structure, on the other hand, allows us also to assign a unique length to a curve or a vector, and to normalise vectors along curves. A spacetime endowed with metric structure is automatically endowed with conformal structure, but not vice versa.

We will now see that the definition of mass-energy-momentum density tensors depends on spacetime structure in a variety of ways; in some cases on its conformal structure, in others on its metric structure. It will turn out that the most general kinds of dependence on spacetime structure, the ones that will turn out to be needed for any energy tensor, for any system to have an energy tensor associated with it and thus be a material system, concern spacetime being endowed with full-blown metric structure.

\subsection{Definitional dependence at the level of the matter fields}

In some cases, the matter fields themselves need to have certain properties in order to allow them to play their role in forming the energy tensor of the material system in question. This is particularly true for all kinds of fluids, where we need the velocity vectors appearing in the respective energy tensor to be of a certain kind. The two simplest fluid systems are 'normal dust' and 'null dust'. The former represents a collection of particles which do not collide with each other (and is hence identical to a pressureless perfect fluid, cf. equation (3)); the latter represents a collection of not directly interacting light rays. The energy tensor of both systems has the form

\footnotetext{
${ }^{36}$ More precisely, conformal structure is given by an equivalence class $\left[g_{\mu \nu}\right]$ such that for any two elements of the class, say $\tilde{g}_{\mu \nu}$ and $\bar{g}_{\mu \nu}$, we have $\tilde{g}_{\mu \nu}=\omega \bar{g}_{\mu \nu}$, where $\omega$ is a smooth, strictly positive function.
} 


$$
T^{\mu \nu}=\rho v^{\mu} v^{\nu} .
$$

In the case of 'normal dust', the velocity vector $v^{\mu}$ has to be time-like, in the case of 'null dust' it has to be light-like. It is these properties that enable the two versions of equation (18) to represent normal or null dust respectively, for a time-like vector field represents an object that moves with less than the speed of light (i.e. a material particle), whereas a light-like vector field represents an object that does move with the speed of light (i.e. a light ray). But these distinctions could not be made if spacetime were not endowed with at least conformal structure, if not with metric structure.

This seems to be all we need to define the energy tensor of null dust. In the case of normal dust, on the other hand, conformal structure is definitely not enough to define the respective energy tensor: we need full-blown metric structure. For an essential part of the model is that the velocity vectors are normalised, $v_{\mu} v^{\mu}=-1$, and in order to normalise vectors, indeed in order to assign them any definite length, we need a metric. ${ }^{37}$

Likewise, for the energy tensors of more complex material systems, we often need metric rather than only conformal structure in order for the matter fields contained in the respective $T_{\mu \nu}$ to have the properties needed. For example, any energy tensor which contains matter fields of the 'same name' but with differing numbers of upper and lower indices (e.g. $F_{\nu}{ }^{\lambda}$ and $F_{\sigma \lambda}$ in the electromagnetic energy tensor (2)) presupposes a unique isomorphism between the tangent space of the manifold and its dual. And we have such an isomorphism only if we have a metric tensor field $g_{\mu \nu}$ defined on the manifold. ${ }^{38}$

I shall call the above dependence of energy tensors on the conformal and/or metric structure of spacetime, namely the need for these structures to be in place in order for the matter fields appearing in the energy tensor to have certain properties, a definitional dependence at the level of the matter fields. We will now see a different kind of dependence, namely the need for spacetime structure in order for the energy tensor itself, as a whole, to have two crucial properties. In fact, a $T_{\mu \nu}$ that lacked these two properties would be judged unphysical.

\footnotetext{
${ }^{37}$ Earman [1989], p.106, makes a similar point, adding that we can distinguish between different amounts of mass-energy of the different dust systems if and only if the velocity vectors are normalised. We will come back to the notion of 'amounts of mass-energy', and the concept of a 'density' linked to it, in section 5.4.

${ }^{38}$ See Wald [1984], pp. 22-5.
} 


\subsection{Definitional dependence at the level of conditions on the energy tensor}

In Newtonian physics, the minimum requirement for a mass density tensor to be regarded as representing a physically possible mass density is that it is positive. Similarly, in relativistic field theory it is demanded that the massenergy density is positive in any frame of reference, for any observer. This constraint is known as the weak energy condition. Malament [2007], p. 240, defines it in the following way:

Weak energy condition: Given any future-directed unit timelike vector $\zeta^{\mu}$ at any point in $M, T_{\mu \nu} \zeta^{\mu} \zeta^{\nu} \geq 0$. [It] asserts that the energy density of [a given matter field], as determined by any observer at any point, is non-negative.

A system for which the weak energy condition does not hold could decay towards a lower and lower energy state without end. ${ }^{39}$

But note that the condition is not even formalisable without a metric. Conformal structure would be enough to define future-directed time-like vectors, but for them to be of unit length we need metric structure. ${ }^{40}$

Could we modify the definition of the weak energy condition in such a way that it would refer only to conformal structure (allowing us to classify a vector as time-like), and not to metric structure (allowing us to assign a vector unit length, or indeed any length at all)? We shall see in the next section that this is not possible, because of the role the vector $\zeta^{\mu}$ plays both here and there.

There is a second property that we demand an energy tensor to possess in order for it to be a physical energy tensor, and we will see that for this demand to be fulfilled the existence of a full-blown metric tensor field is needed. The standard definition of this constraint is as follows (see again Malament [2007], p.240):

\footnotetext{
${ }^{39}$ The importance of this condition can be seen in the debate about the two mathematical representations of Brans-Dicke theory, the Jordan frame and the Einstein frame of the theory. The details of these representations are not important here; what is important is the army of physicists who claim that the Jordan frame is 'unphysical' because it allegedly violates the weak energy condition. See for example Faraoni and Gunzig [1999], who argue this point.

${ }^{40}$ The same is true for the dominant energy condition, for the same reason; see Malament [2007], p.240, for the definition.
} 
Conservation condition: $\nabla^{\mu} T_{\mu \nu}=0$ for all points in spacetime. [It] asserts that the energy-momentum carried by [a matter field] is locally conserved.

Note that the conservation equation is an automatic consequence of the Einstein field equations, and indeed in a Lagrangian formulation it is a consequence of both the gravitational and the matter field equations. ${ }^{41}$ More importantly, all empirical data we have strongly suggests that this condition holds, i.e. that mass-energy-momentum is never created or destroyed but only ever transferred. ${ }^{42}$

However, the conservation condition would not in general hold for a connection that is derived from a merely conformal metric, i.e. one which is not uniquely defined but allows for the conformal transformation $\tilde{g}_{\mu \nu}=\omega \bar{g}_{\mu \nu}$. Furthermore, if we assume that equation (5.2) holds, the conformal factor $\omega$ is determined, and conformal structure is extended to metric structure. ${ }^{43}$

Thus, we may be willing to consider that new data might come along that shows that the conservation equation does not hold for every system with an associated $T_{\mu \nu}$ - but the point is that we could not even judge whether the equation holds or fails in general for that system if we had only conformal rather than full-blown metric structure.

In sum, we see that we need spacetime to be endowed with a metric tensor field in order to define the mass-energy-momentum tensors of material systems in a way that leaves any hope of their representing the properties of actual material systems.

\subsection{Abstract definitional dependence}

A fourth kind of dependence on the metric becomes evident if we look at $T_{\mu \nu}$ in the most abstract manner: as a map. Indeed, we can see $T_{\mu \nu}$ as a machine that always gives us the mass-energy density of a given system at a given point $p$ relative to the state of motion of an arbitrary observer (at $p$ ), whose 4 -velocity is represented by a future-directed unit-timelike vector $\zeta^{\mu}$ (at $p$ ).

\footnotetext{
${ }^{41}$ See for example Brown and Brading [2002].

${ }^{42}$ Strictly speaking the conservation condition only gives a conservation law in the classical sense if the metric with which $\nabla^{\mu}$ is compatible admits a Killing vector field. For only then can $\nabla^{\mu} T_{\mu \nu}=0$ be given an integral form, which can then be interpreted as saying that the total mass-energy-momentum flux over a closed surface is zero. See e.g. Hawking and Ellis [1973], p.62, for details.

${ }^{43}$ Cf. Hawking and Ellis [1973], p.63.
} 
The mass-energy-momentum tensor is then a map $T: T_{p} M \times T_{p} M \rightarrow \Theta$, where $T_{p} M$ is the tangent vector space $\zeta^{\mu}$ lives in, and $\Theta$ is the set of all possible mass-energy densities $\rho$. In particular, if $T_{\mu \nu}$ is the energy tensor of an arbitrary material system observed by an observer with the 4 -velocity $\zeta^{\mu}$ at point $p$, then $T_{\mu \nu} \zeta^{\mu} \zeta^{\nu}$ is the mass-energy density of the system as measured by that observer. ${ }^{44}$

Note that in order to be capable of representing the state of motion of an observer, the 4-vector $\zeta^{\mu}$ must be time-like, so that it can only represent sub-luminal motion. Furthermore, it needs to have a definite magnitude, so that we can distinguish between observers with different states of motion (including them having 3-velocities of different magnitude) in the first place. And if we can assign a definite magnitude to $\zeta^{\mu}$, then we can also normalise it, i.e. make it a vector of unit length $\left(\zeta^{\mu} \zeta_{\mu}=-1\right)$.

In order to define $\zeta^{\mu}$ as time-like, conformal structure would be sufficient. But conformal structure does not allow to determine the lengths of vectorsin order to do that, we need metric structure.

This is where a definitional dependence of $T_{\mu \nu}$ on metric structure comes in yet again, this time in as general a fashion as possible. If we did not have a metric tensor field $g_{\mu \nu}$, then the map $T: T_{p} M \times T_{p} M \rightarrow \Theta$ would not allow us to distinguish between the mass-energy-momentum density as measured by different observers. One could thus say that we need abstract definitional dependence of $T_{\mu \nu}$ on $g_{\mu \nu}$ if we do not want to have to give up pursuing relativity theory altogether!

\subsection{Interpretational dependence}

I call the fifth kind of dependence of energy tensors on spacetime structure an 'interpretational dependence' because arguably we need it in order to interpret $T_{\mu \nu}$ as a mass-energy-momentum density in the first place. In particular, we need a volume element, with the help of which we can speak of the density in a particular volume. If we did not have a volume element, we could not distinguish between different amounts of mass-energy-momentum in different parts of a material system, nor compare the amounts of mass-energymomentum possessed by different material systems. For we can obtain the amount of mass-energy-momentum of a material system only by integrating the density over the volume occupied by the system, and only if we can do

\footnotetext{
${ }^{44}$ For this way of defining the energy-momentum tensor see e.g. Malament [2007], p.240.
} 
that does it make sense to speak of $T_{\mu \nu}$ as a density in the first place. ${ }^{45}$

However, the natural volume element of spacetime is defined in terms of a metric tensor. ${ }^{46}$ But could conformal structure suffice to define the volume element needed? No, because for every metric in the equivalence class of conformally equivalent metrics we will have a different volume element, and integrating over different volume elements for different material systems (or different parts of the same system) would rob us of the ability to compare the amounts of mass-energy-momentum they possess. Thus, we need a unique metric tensor to give us a unique volume element, and hence we end up having yet another kind of dependence of energy tensors on metric structure.

This form of dependence can even be seen to apply to a Newtonian mass density, indeed to all kinds of densities. Newtonian mass density is represented by a scalar field $\rho$, and the definition of a scalar field as such - it hardly need be said - does not depend on the metric. But similar to what we noted about amounts of mass-energy above, the amount of mass of an extended Newtonian object can be properly defined only via integrating the mass density over a (Euclidean) volume element, and for that, in turn, we need a metric. This is true even for the proper definition of the total mass of a point particle, where the integration process involves use of an appropriate delta function. ${ }^{47}$

We have encountered five kinds of dependence of energy tensors on the metric field. The first was a functional dependence of energy tensors on the metric, the kind of dependence we found not to hold in general and to be only the tip of the iceberg. We then saw that there are three different kinds of definitional dependence of energy tensors on the metric.

\footnotetext{
${ }^{45}$ Earman [1989], p.106, makes a similar point, although for the sake of simplicity he discusses only the case of (normal) dust.

${ }^{46}$ More precisely, given a metric $g_{\mu \nu}$ and a normalisation condition, there is a unique (up to a sign, i.e. up to a choice of orientation) volume element $d \Omega$ determined by $g_{\mu \nu}$, namely $d \Omega=\sqrt{-g} d x^{1} \wedge d x^{2} \wedge d x^{3} \wedge d x^{4}$. See Wald [1984], pp.432-3, for details.

${ }^{47}$ I said at the beginning of the article that the claim that at least in Newtonian physics mass is an intrinsic property of material systems is initially plausible. The reason is that one often thinks of mass as a primitive property, one not defined in terms of others. However, it should be noted that Newton's own conception of mass was not of this kind, that indeed one could argue that Newton's primary conception of mass (cf. footnote 9), commits him to a position quite like the one outlined in the above paragraph. For Newton defines mass as a measure of matter, a measure "that arises from its density and volume jointly" (Definition 1 of Newton [1999]).
} 
The first concerned Lagrangian theories, where we found that matter fields have to couple directly to the metric field in order for an energy tensor to be definable.

The second was a frequent, but not general, definitional dependence at the level of the matter fields making up specific energy tensors: we found that in many cases the matter fields featuring in the energy tensor need a metric field in place in order appropriately to represent the properties of the material system in question. However, we also found that in some particularly simple systems, conformal structure, i.e. an equivalence class of metrics, may be sufficient to fulfil this task.

Thirdly, we found a general definitional dependence at the level of the energy conditions. In particular, we looked at the weak energy condition and the conservation condition. Any energy tensor failing to satisfy either of these conditions would reasonably be regarded as unphysical; but in order for a $T_{\mu \nu}$ to fulfil them, spacetime needs to be endowed with a metric tensor field.

The fourth kind of definitional dependence of $T_{\mu \nu}$ on $g_{\mu \nu}$ I termed abstract definitional dependence: we need the metric in order to define $T_{\mu \nu}$ as a map from pairs of unit timelike 4-vectors to energy densities, which seems the most general definition of $T_{\mu \nu}$ one could give.

Finally, we even found an interpretational dependence of $T_{\mu \nu}$ on $g_{\mu \nu}$, pointing out that a metric field is needed in order to interpret tensor fields as representing physical densities in the first place. ${ }^{48}$

It follows that the energy tensor $T_{\mu \nu}$ is not a 'source' of the metric field in the same way as the mass density $\rho_{m}$ is a source of the gravitational field in Newtonian theory, or as the electric charge density $\rho_{e}$ is a source in electrostatics. In both cases, one can specify the source first and then obtain the gravitational or the electric field, respectively, via the field equations. This is not possible for the energy tensor $T_{\mu \nu}$, whose very definition already depends on the field $g_{\mu \nu}$ for which it is supposed to act as a source. ${ }^{49}$

\footnotetext{
${ }^{48}$ Note that in all these cases the situation did not depend on whether the metric was a static or a dynamical field.

${ }^{49}$ Wald [1984], p.73, makes a similar point, although he gives as a reason only the explicit dependence of energy tensors on $g_{\mu \nu}$ we mentioned in section 3. One might also claim that in GR the metric field itself can act as a source because the non-fundamental 'source field' $T_{\mu \nu}$ depends on $g_{\mu \nu}$. This could then be seen as the cause of the non-linearity of the field equations, i.e. the failure of a superposition principle to hold for the solutions of the Einstein field equations. However, one has to be careful: even the vacuum Einstein
} 
But what does this tell us with respect to the question of whether massenergy-momentum density is an intrinsic or extrinsic property of material systems?

\section{What kind of property is mass-energy-momentum density?}

In section 1, I pointed out that the only thing that would really answer the question of which was more fundamental, matter or spacetime structure, would be if one was a requirement for the existence of the other but not vice versa. ${ }^{50}$ But the tensor field that is supposed to represent the main properties of matter, the mass-energy-momentum density tensor $T_{\mu \nu}$, is a non-fundamental field, and it does require both the metric field $g_{\mu \nu}$ and the matter fields $\Phi$ in order to be defined, and in order to have some of its crucial properties.

What kind of property is thus represented by $T_{\mu \nu}$ ?

\subsection{A relational property?}

Lewis [1983], pp.111-2, introduces the distinction between intrinsic and extrinsic properties in the following way:

A sentence or statement or proposition that ascribes intrinsic properties to something is entirely about that thing; whereas an ascription of extrinsic properties to something is not entirely about that thing, though it may well be about some larger whole which includes that thing as part. A thing has its intrinsic properties in virtue of the way that thing itself, and nothing else, is. Not so for extrinsic properties, though a thing may well have these in virtue of the way some larger whole is. The intrinsic

equations are non-linear, and so even here the metric field can act as 'a source for itself'. Thus, it seems that in GR the clear distinction between a field and its source as we have it in electrodynamics gets questioned on more than one front, even though the fronts are interconnected.

${ }^{50}$ Note that $A$ requiring $B$ while $B$ not requiring $A$ is sufficient, but not necessary, for $B$ to be more fundamental than $A$. For if $A$ did not require $B$ but $C$, while $B$ required neither $A$ nor $C$ (nor anything else), then $B$ would also be more fundamental than $A$. 
properties of something depend only on that thing; whereas the extrinsic properties of something may depend, wholly or partly, on something else.

We have seen in sections 4 and 5 that the definition of energy tensors depends not only on the matter fields $\Phi$, but also on spacetime structure; in some ways on its conformal structure $\left[g_{\mu \nu}\right]$ but more generally on its metric structure $g_{\mu \nu}$.

It seems to follow that the energy tensor $T_{\mu \nu}$ must be seen as corresponding to an extrinsic property of material systems. For matter only has an energy tensor $T_{\mu \nu}$ associated with it in virtue of the relations holding between the material system (whose fundamental properties are represented by the matter fields $\Phi$ ) and the geometric structure of spacetime.

We should now distinguish between relations and relational properties, in order to find out what kind of extrinsic property exactly we should see $T_{\mu \nu}$ to be, and to see what it is a property of.

An $n$-place relation $G\left(x_{1}, \ldots, x_{n}\right)$ depends on $n$ entries. An example of a 2-place relation is ' $R x y:=(x$ is the father of $y)$ '. A relation gives rise to a set of relational properties. For example, Hermann Einstein and Albert Einstein stand in certain relations to each other, which give rise to Hermanns relational property of being a father, and to Albert's relational property of being a son.

We can make this distinction more precise in the following way: let us call an $n$-place predicate, where $n \geq 2$, a relation if and only if it contains more than one free variable $x, y, z$, while it is allowed to contain an arbitrary number of designators $a, b, c$. Let us speak of a relational property if we have an $n$-place predicate that contains at most one free variable, and at least one designator.

Then we can think of Hermann and Albert Einstein in the following way. We can think of them as two individuals (or two systems each of which consists of only one individual) who stand in certain relations to each other and have hence various relational properties. For example, if we denote the (asymmetric) relation ' $x$ is a son of $y$ ' as $S x y$, Albert as $a$ and Hermann as $h$, then the fact that Albert possesses the relational property of 'being the son of someone' can be expressed by the sentence $\exists y S a y$, while the fact that Albert possesses the relational property of 'being the son of Hermann Einstein' can be expressed by the sentence $S a h$. Both properties are properties of Albert only, even though they are due to his standing in a certain relation 
to Hermann.

Rather than looking at the system consisting only of Albert Einstein and wondering about the relational properties he has in virtue of standing in a certain relation to his father, we could also look at the bigger system (Albert, Hermann $)=(a, h)=: s$ and wonder about the properties the system $s$ has in virtue of the relations its parts stand in. One intrinsic property $s$ has (a property that only depends on $s$ itself, but not on the relation $s$ has to anything else) is that its elements stand in a father-son relationship. Let us denote this 1 -place property of systems by $F x$, and the fact that $s$ possesses this intrinsic property as being expressed by the sentence $F s$.

But not much seems to depend on whether we choose the first or the second perspective. In both cases we can say everything we want to say about Hermann and Albert Einstein in this context, and in both cases the important fact is that the relation between the two leads to certain systems possessing certain properties. Still, it is an interesting question whether the energy tensor $T_{\mu \nu}$ is more like Albert's relational property of having a father, $\exists y F a y$ (case 1), or whether it is more alike the property of a system like (Albert, Hermann) that its parts stand in a father-son relationship, $\exists x \exists y F x y$ (case 2).

If the latter is the case, we should regard the predicate $\mathscr{T}_{1}(x)$, where the domain of $x$ is 'systems in which both a metric field $g_{\mu \nu}$ and at least one matter field $\Phi$ is defined', as expressing the intrinsic property of such systems to have an energy tensor (rather than one with particular components) associated with them, a property they have because of the relation(s) that hold between the material system and spacetime structure.

If the former is the case, we should say that possession of some massenergy-momentum density $T_{\mu \nu}$ is a property of only the material parts of the total system, which are represented by the matter fields $\Phi$, a property that the material system has in virtue of its relations to the metric field $g_{\mu \nu}$. We would then say that although $T_{\mu \nu}$ depends on the relations the matter fields $\Phi$ have to the metric field $g_{\mu \nu}$ (like Albert's property of being the son of Hermann depends on his relations to Hermann), the property 'possessing mass-energy-momentum' is still a property only of matter, just as it is a (relational) property of only Albert that he is the son of Hermann. We can reformulate this by calling $\mathscr{T}_{2}(x, y)$, where the domain of $x$ is 'metric fields' and the domain of $y$ is 'matter fields', the relation which allows us to express the fact that a material system represented by $\Phi$ has the relational property of having an energy tensor associated with it. The trouble with this is only 
that nothing in the representation $\mathscr{T}_{2} g_{\mu \nu} \Phi$ gives away that $\mathscr{T}_{2}$ is a property of the part of the total system represented by $\Phi$ only - but that is also the case for using $S a h$ in order to represent system Albert's property of being system Hermann's son.

As in the case of Hermann and Albert, I do not think that much depends on the perspective we choose. Indeed, one could arguably represent both $\mathscr{T}_{1}$ and $\mathscr{T}_{2}$ as a map from the set of pairs $\left(g_{\mu \nu}, \Phi\right)$ to the set of all energymomentum tensors, $\mathscr{T}:\left(g_{\mu \nu}, \Phi\right) \longrightarrow T_{\mu \nu}\left(g_{\mu \nu}, \Phi\right){ }^{51}$ The distinction would merely lie in seeing the set $\left(g_{\mu \nu}, \Phi\right)$ as an 'object' or as a pair of objects. Anyhow, we find that material systems possessing (non-vanishing) massenergy-momentum tensors depends on a relation between the material system and the structure of spacetime. ${ }^{52}$

\subsection{Relational and essential?}

In section 2.2 I have argued that we treat possession of mass-energy-momentum as an essential property for a system to count as a material system. Now we have seen in the previous section that it is also a relational property, a property material systems only have in virtue of their relation to spacetime structure.

It might be claimed that every essential property must be an intrinsic property. Indeed, the claim can be argued to follow from Lewis' principles

\footnotetext{
${ }^{51}$ Of course, this is only the simplest case: if a system contains $m$ matter fields, then $\mathscr{T}$ is an $m+1$-relation: $\mathscr{T}:\left(g_{\mu \nu}, \Phi_{1}, \ldots, \Phi_{m}\right) \longrightarrow T_{\mu \nu}\left(g_{\mu \nu}, \Phi_{1}, \ldots, \Phi_{m}\right)$.

${ }^{52}$ Note: there remains the age-old question of whether the metric/gravitational field could possess energy-momentum in its own right, and in the same way as the matter fields. Given what I have argued above, the success of such a project would call into question the very distinction between 'matter fields' like $\Phi$ on the one hand and 'geometric fields' like $g_{\mu \nu}$ on the other, and hence be an important step towards a unified field theory. But alas, at least within GR, we have only the choice between associating a coordinatedependent pseudo-tensor with the energy of the gravitational field, to restrict our attention to asymptotically flat spacetimes, or to accept that the energy of a gravitational field is a fundamentally non-local quantity. So even if we do go down the road of thinking of GR as telling us that gravitational fields possess energy-momentum as well, they still do so in such a way that a fundamental distinction between two different kinds of fields - matter fields and metric field - remains. For a historic discussion of the debate between Einstein, Lorentz and Levi-Civita on the first possibility above (pseudo-tensors) see Cattani and DeMaria [1993]; for a recent discussion of the second possibility (asymptotically flat spacetimes) see Poisson [2004], especially section 4.3.
} 
of recombination and plenitude for the creation of possible worlds, if recombination is restricted to natural properties. ${ }^{53}$

On the other hand, the proposal that relational properties can be possessed essentially is not new either. Already Moore [1922] wrote: ${ }^{54}$

Let $P$ be a relational property, and $a$ a term to which it does in fact belong. I propose to define what is meant by saying that $P$ is internal to $a$ (in the sense we are now concerned with) that from the proposition that a things [sic] has not got $P$, it 'follows' that it is other than a.

One famous candidate for an essential relational property, arising from an internal relation, has been proposed by Kripke, and it goes under the name of 'the essentiality of origin'. Kripke argues that it is an essential property of every person, i.e. a property that the respective person has in every possible world, that they have the same parents they actually have (Kripke [1972], p.113):

How could a person originating from different parents, from a totally different sperm and egg, be this very woman? One can imagine, given the woman, that various things in her life could have changed [...]. But what is harder to imagine is her being born of different parents. It seems to me that anything coming from a different origin would not be this object.

And of course, the property of having the parents one has is a relational property, making it an essential relational property every person has. Let us

\footnotetext{
${ }^{53} \mathrm{Cf}$. Lewis [1986]. The principle of recombination states that it is possible to recombine the natural properties (such as charge, mass, colour) of the actual world in order to get a new possible world. The principle of plenitude states that there actually is a possible world for every recombination of the natural properties of our world. The principle of recombination then implies that a relation that holds in our world must not hold in every possible world (there is a possible world in which Hermann Einstein does not have a son), and the principle of plenitude implies that for every recombination there is indeed a possible word arising from it. So there is no relation, and hence no relational property, that holds true of the same object in every possible world. Hence, both principles together with our definition of what an essential property of an object is (a property the respective object could not lack) imply that every essential property must be an intrinsic property.

${ }^{54}$ Dunn [1990] distinguishes different senses of 'internal relation' with the help of his relevance logic, but I shall not introduce them here.
} 
accept Kripke's assertion of the essentiality of origin as a working hypothesis for now. Of course, there is an asymmetry here: even if one thinks that it is an essential relational property of Albert that he is Hermann Einstein's son, this does not necessarily mean that it is also an essential relational property of Hermann that he is Albert's father. In another world, Hermann might not have had children, and still be the same Hermann Einstein, whereas, according to Kripke, it is not possible for Albert to have another father than Hermann and still be the same Albert Einstein.

Anyhow, note that the claim that mass-energy-momentum is an essential relational property of matter is a much weaker claim than Kripke's. There is a crucial difference between claiming that every person essentially has a particular pair of people as parents, and claiming that every material system essentially possesses some mass-energy-momentum density.

So our case corresponds to a weaker version of Kripke's original claim, which one could formulate as follows: In order for $\Phi$ to be a human being, he/she needs to have some parents, but not a particular pair. Similarly, in order for $\Phi$ to be a matter field, it needs to have some mass-energy-momentum density tensor $T_{\mu \nu}$ associated with it. The structure of its energy tensor $T_{\mu \nu}$ tells us only what kind of matter a given matter field $\Phi$ can describe- for example, we need vanishing diagonal components for an electromagnetic field and non-vanishing diagonal components for a perfect fluid - but in order to know that it is a matter field in the first place, we only need to know that there is an energy tensor associated with the field.

\section{Conclusion}

I started out by describing how Newton's defining property of material systemsthe property of possessing mass - was generalised by relativistic field theory to the requirement that such systems need to have a mass-energy-momentum density tensor $T_{\mu \nu}$ associated with them.

We have then seen that the definition of energy tensors depends on the metric field in a variety of ways. Hence, in relativistic field theory, massenergy-momentum cannot be regarded as an intrinsic property of matter, but must be seen as a relational property of matter (or a property of systems containing matter) that it has only because of its relation to spacetime structure.

Is it a surprising result that $T_{\mu \nu}$ does not describe an intrinsic property 
of matter? As already mentioned in the introduction, nobody would have claimed that the momentum of a particle in Newtonian mechanics was an intrinsic property of this particle; it is a property that makes sense only if we describe that particle as changing its position with respect to something else. Kinetic energy too might be regarded as a relational property in Newtonian mechanics, for it depends on the velocity of the particle, which is a relational property for the same reason as the momentum of the particle. And even mass density can be argued to be not an intrinsic property of material systems, although the dependence of mass density on spacetime structure is surely of a weaker kind than that of relativistic mass-energy-momentum density tensors. Arguably, all this is grist to the mill of certain structural realists, who think that there are no intrinsic properties whatsoever, or that we can at least have no knowledge about them. ${ }^{55}$

In the introduction, I mentioned that the early Einstein was strongly motivated by a version of Mach's principle when he created GR, a version in which he claimed the geometric field $g_{\mu \nu}$ should be determined by the energy tensor $T_{\mu \nu}$. Einstein's idea was that spacetime structure should be derived from the properties of material systems. We have seen that, on the contrary, the very definition of energy tensors depends on spacetime structure, and that hence even a unique determination of $g_{\mu \nu}$ by $T_{\mu \nu}$ would not be sufficient for spacetime geometry to be reduced to the properties of matter.

But nor does GR accord to what might be called an Anti-Machian principle: even though the matter fields $\Phi$ do not determine the spacetime structure $g_{\mu \nu}$, spacetime does not determine the material structures either. However, matter needs spacetime in order to have some of its key properties defined, while spacetime does not depend on matter in this way. Still, in order to get a truly Anti-Machian theory, we would need not only the energy tensor to depend on the metric field, but the matter fields themselves would need to be derivable from the structure of spacetime. One candidate for such a theory is Kaluza-Klein theory, in which the electromagnetic vector potential forms part of the 5-dimensional metric tensor, and hence leads to the electromagnetic field $F_{\mu \nu}$ itself being derivable from the geometric properties of spacetime.

But this leads us too far afield. The energy-momentum tensor of matter depends on, but is not determined by, the structure of spacetime - and that

\footnotetext{
${ }^{55}$ See Ladyman [2009] for a classification of the different versions of structural realism, alongside an extensive bibliography.
} 
is enough for today.

\section{Funding}

This work was supported by the British Society for the Philosophy of Science, who granted me a Doctoral Scholarship without which this work would not have been possible. Further support came from the Arts and Humanities Research Council, Oriel College, Oxford University, and the Einstein Papers Project at the California Institute of Technology. I wish to express my profound thanks.

\section{Acknowledgements}

I am very grateful to Oliver Pooley, Harvey Brown, Jeremy Butterfield, Eleanor Knox, Carl Hoefer, David Wallace, Robert Geroch, Stephen Lyle, Tilman Sauer, Erhard Scholz, Edward Slowik and Stephen Tiley. Each of them read one or more than one version of this paper, and helped improve it significantly with their comments and suggestions. I wish to thank Oliver Pooley particularly, who showed endless patience in reading what must have felt like 2 dozen different versions of the paper - and it may actually have been that many. Any remaining errors are of course solely mine.

I also thank Robert Geroch, Erik Curiel, Stephen Lyle and John Norton for very helpful discussions, in particular about the energy tensors of different fluid systems and their relation to spacetime structure, and David Malament and Erik Curiel for enlightening discussions about the more subtle ways in which energy tensors depend on spacetime structure. Furthermore, I am grateful to Eric Schliesser for a very helpful exchange about Newton's conception of mass.

Last but not least, I am grateful to audiences in Oxford, Madrid, Montreal, St. Andrews and Irvine for helpful and fascinating debates after having presented this paper at various stages of its development.

\section{References}

Barbour, J. [2007], Einstein and Mach's principle, in J. Renn and M. Schemmeltth, eds, 'The Genesis of General Relativity', Vol. 3, Springer. 
Barbour, J. and Pfister, H., eds [1995], Mach's Principle. From Newton's Buckett to quantum Gravity, Vol. 6 of Einstein Studies, Birkhäuser.

Belinfante, F. [1940], 'On the current and the density of the electric charge, the energy, the linear momentum and the angular momentum of arbitrary fields', Physica vii, 449-474.

Biener, Z. and Smeenk, C. [forthcoming], Cotes' queries: Newton's empiricism and conceptions of matter, in A. Janiak and E. Schliesser, eds, 'Interpreting Newton', Cambridge University Press, Cambridge.

Brading, K. A. and Ryckman, T. A. [2008], 'Hilbert's 'Foundations of Physics': Gravitation and Electromagnetism within the axiomatic method', Studies in History and Philosophy of Modern Physics 39(102-153).

Brown, H. R. [2005], Physical Relativity. Space-time structure from a dynamical perspective, Oxford University Press.

Brown, H. R. and Brading, K. A. [2002], 'General Covariance from the perspective of Noether's Theorems', Diálogos 79, 59-86.

Brown, H. R. and Pooley, O. [2004], Minkowski space-time: a glorious non-entity, in D. Dieks, ed., 'The Ontology of Spacetime', Vol. I of Philosophy and Foundations of Physics, Elsevier.

Cao, T. Y. [1997], Conceptual developments of 20th century field theories, Cambridge University Press.

Cattani, C. and DeMaria, M. [1993], Conservation laws and gravitational waves in general relativity (1915-1918), in J. Earman, M. Janssen and J. Norton, eds, 'The Attraction of Gravitation: New Studies in the History of General Relativity', Vol. 5 of Einstein Studies, Birkhäuser.

Corry, L. [1999], 'From Mie's electromagnetic theory of matter to Hilbert's unified foundations of physics', Studies in History and Philosophy of Modern Physics 30B(2), 159-183.

DeFelice, F. and Clarke, C. [1990], Relativity on Curved Manifolds, Cambridge Monographs on Mathematical Physics, Cambridge University Press.

Doughty, N. A. [1990], Lagrangian Interaction, Perseus Books.

Dunn, J. M. [1990], 'Relevant predication 2: Intrinsic properties and internal relations', Philosophical Studies 60, 177-206.

Earman, J. [1989], World Enough and Space-Time: Absolute versus Relational Theories of Space and Time, MIT Press, Cambridge MA.

Einstein, A. [1905a], 'Zur Elektrodynamik bewegter Körper', Annalen der Physik 17, 891921. Reprinted as Document 23 (page 275) of Volume 2 CPAE. 
Einstein, A. [1905b], 'Ist die Trägheit eines Koerpers von seinem Energieinhalt abhängig?', Annalen der Physik 18, 639-641. Reprinted as Document 24 (page 311) of Volume 2 CPAE.

Einstein, A. [1914], 'Die formale Grundlage der allgemeinen Relativitätstheorie', Sitzungsberichte der Königlich Preussischen Akademie der Wissenschaften pp. 799-801. Reprinted as Document 9 (page 72) of Volume 6 CPAE.

Einstein, A. [1916], 'Hamiltonsches Prinzip und allgemeine Relativitätstheorie', Sitzungsberichte der Königlich Preussischen Akademie der Wissenschaften pp. 1111-1116. Reprinted as Document 41 (page 409) of Volume 6, CPAE.

Einstein, A. [1918], 'Prinzipielles zur allgemeinen Relativitätstheorie', Annalen der Physik 55, 241-244. Reprinted as Document 4 (page 37) of Volume 7 CPAE.

Einstein, A. and Grossmann, M. [1914], 'Kovarianzeigenschaften der Feldgleichungen der auf die verallgemeinerte Relativitätstheorie gegründeten Gravitationstheorie', Zeitschrift für Mathematik und Physik 63, 215-225.

Faraoni, V. and Gunzig, E. [1999], 'Einstein frame or Jordan frame?', International Journal for Theoretical Phyiscs 38(217).

Flores, F. [2005], 'Interpretations of Einstein's equation $E=m c^{2}$ ', International Studies in the Philosophy of Science 19(3), 245-260.

Giulini, D. [2008], 'What is (not) wrong with scalar gravity?', Studies in the History and Philosophy of Modern Physics 39, 154-180.

Goenner, H. F. M. [1984], 'Theories of gravitation with nonminimal coupling of matter and the gravitational field', Foundations of Physics 14(9), 865-881.

Goenner, H. F. M. [1996], Einführung in die spezielle und allgemeine Relativitätstheorie, Spektrum, Akademischer Verlag.

Hall, G. S. [2004], Symmetries and Curvature Structure in General Relativity, Vol. 46 of World Scientific Lecture Notes in Physics, World Scientific.

Hawking, S. and Ellis, G. [1973], The Large Scale Structure of Space-Time, Cambridge Monographs on Mathematical Physics, Cambridge University Press.

Hilbert, D. [1915], 'Die Grundlagen der Physik', Königliche Gesellschaft der Wisenschaften zu Göttingen, Nachrichten . Part I: (1915), pp.395-407; Part II: (1916), pp.53-76.

Hobson, M., Efstathiou, G. and Lasenby, A. [2006], General Relativity: An Introduction for Physicists, Cambridge University Press.

Hoefer, C. [1994], 'Einstein's struggle for a Machian gravitation theory', Studies in History and Philosophy of Science 25(3), 287-335. 
Janssen, M. [2007], 'The line between kinematics and dynamics in special relativity', PhilSci Archive: 00003895 .

Janssen, M. and Mecklenburg, M. [2007], From classical to relativistic mechanics: Electromagnetic models of the electron, in V. Hendricks, K. Jorgensen, J. Lützen and S. Pedersen, eds, 'Interactions: Mathematics, Physics and Philosophy, 1860-1930', Dordrecht: Springer.

Kichenassamy, S. [1993], Variational derivations of Einstein's equations, in M. J. John Earman and J. Norton, eds, 'Einstein Studies Volume 5: The Attraction of Gravitation: New Studies in the History of General Relativity.', Birkhäuser.

Kripke, S. [1972], Naming and Necessity, Harvard University Press.

Ladyman, J. [2009], Structural realism, in 'The Stanford Encyclopedia of Philosophy', Edward N. Zalta.

URL: http://plato.stanford.edu/archives/sum2009/entries/structural-realism/

Lanczos, C. [1986], The Variational Principles of Mechanics, Dover.

Landau, L. and Lifschitz, E. [1966], Fluid Mechanics, Vol. 6 of Course of Theoretical Physics, Pergamon Press.

Landau, L. and Lifschitz, E. [1989], The classical theory of fields, Vol. 2 of Course of Theoretical Physics, Oxford: Pergamon.

Lange, M. [2001], 'The most famous equation', The Journal of Philosophy 98(5), 219-238.

Lange, M. [2002], An Introduction to the Philosophy of Physics: Locality, Fields, Energy and Mass, Wiley Blackwell.

Leclerc, M. [2006], 'Canonical and gravitational stress-energy tensors', arXiv:grqc/0510044v625 Aug 2006 .

Lewis, D. [1983], 'Extrinsic properties', Philosophical Studies 44, 197-200.

Lewis, D. [1986], On the Plurality of Worlds, Blackwell Publishing.

Malament, D. [2007], Classical relativity theory, in J. Butterfield and J. Earman, eds, 'Philosophy of Physics', Vol. A, Elsevier.

Minkowski, H. [1908a], 'Raum und Zeit', Talk given at the 80th Tagung deutscher Naturforscher und Ärzte in Cologne, 21.9.1908 . Reprinted in Lorentz, Einstein, Minkowski 1982.

Minkowski, H. [1908b], 'Die Grundgleichungen für die elektromagnetischen Vorgänge in bewegten Körpern', Nachrichten der Königlichen Gesellschaft der Wissenschaften zu Göttinngen, Mathematisch-Physikalische Klasse pp. 53-111. Reprinted in: Gesammelte Abhandlung von Hermann Minkowski, Vol.2, Leipzig, 1911, 352-404. 
Moore, G. [1922], 'External and internal relations', Philosophical Studies pp. 253-275.

Narlikar, J. [1993], Introduction to Cosmology, Cambridge University Press.

Newton, I. [1962], De gravitatione, in A. Hall and M. Hall, eds, 'Unpublished Scientific Papers of Isaac Newton', Cambridge University Press, Cambridge.

Newton, I. [1999], The Principia, Mathematical Principles of Natural Philosophy: A New Translation, 3 edn, University of California Press, Berkeley. Translated by I. Bernard Cohen and Anne Whitman, 1999.

Norton, J. [1992], 'Einstein, Nordström and the early demise of Lorentz-covariant, scalar theories of gravitation', Archive for History of Exact Sciences 45, 17-94.

Norton, J. [2008], 'Why constructive relativity fails', British Journal for the Philosophy of Science 59, 821-834.

Ohanion, H. C. [2008], 'Did Einstein prove $E=m c^{2}$ ?', Studies in History and Philosophy of Modern Physics 40, 167-173.

Poisson, E. [2004], A Relativist's Toolkit: The Mathematics of Black-Hole Mechanics, Cambridge University Press.

Renn, J. [2007], The third way to general relativity: Einstein and Mach in context, in J. Renn and M. Schemmel, eds, 'The Genesis of General Relativity', Vol. 3 of Boston Studies in the Philosophy of Science, Springer.

Robertson, T. [2008], Essential vs. accidental properties, in 'The Stanford Encyclopedia of Philosophy', Edward N. Zalta.

URL: http://plato.stanford.edu/archives/sum2008/entries/essential-accidental/

Rosenfeld, L. [1940], 'Sur le tenseur d'impulsion-energie', Mem. Roy. Acad. Belg. Cl. Sci. $18,(6)$.

Saraví, R. E. G. [2002], 'The electromagnetic energy-momentum tensor', J.Phys A35, 9199-9204.

Sauer, T. [1999], 'The relativity of discovery: Hilbert's first note on the foundations of physics', Archive for History of Exact Sciences 53, 529-575.

Sauer, T. and Majer, U. [2005], Hilbert's "world equations" and his vision of a unified science, in J. A.J.Kox, ed., 'The Universe of General Relativity', Vol. 11 of Einstein Studies, Birkhaeuser, pp. 259-276.

Smeenk, C. and Martin, C. [2007], Mie's theories of matter and gravitation, in J. Renn and M. Schemmel, eds, 'The Genesis of General Relativity', Vol. 4 of Boston Studies in the Philosophy of Science, Springer. 
Vizgin, V. [1994], Unified Field Theories in the first third of the 20th century, Birkhäuser. von Laue, M. [1911a], Das Relativitätsprinzip, Friedrich Vieweg und Sohn.

von Laue, M. [1911b], 'Zur Dynamik der Relativitätstheorie', Annalen der Physik 35, 524542.

Wald, R. M. [1984], General Relativity, The University of Chicago Press, Chicago and London.

Weatherson, B. [2007], Intrinsic vs. extrinsic properties, in E. N. Zalta, ed., 'The Stanford Encyclopedia of Philosophy'.

URL: http://plato.stanford.edu/archives/spr2007/entries/intrinsic-extrinsic/

Weinstein, S. [1996], Strange couplings and space-time structure, Vol. 63, Supplement, Philosophy of Science Association.

Wien, W. [1900], 'Ueber die Möglichkeit einer elektromagnetischen Begründung der Mechanik', Arch. neèrl. 5. Reprinted in Annalen der Physik Volume 5 (1901), pp.501-3. Page references refer to this reprint. 